\title{
Transit Detection of Radial Velocity Planets
}

\author{
Stephen R. Kane and Kaspar von Braun
}

\author{
Michelson Science Center, Caltech, MS 100-22, 770 South Wilson Avenue Pasadena, CA \\ 91125, USA \\ email: skane@ipac.caltech.edu
}

\begin{abstract}
.
The orbital parameters of extra-solar planets have a significant impact on the probability that the planet will transit the host star. This was recently demonstrated by the transit detection of HD 17156b whose favourable eccentricity and argument of periastron dramatically increased its transit likelihood. We present a study which provides a quantitative analysis of how these two orbital parameters affect the geometric transit probability as a function of period. Further, we apply these results to known radial velocity planets and show that there are unexpectedly high transit probabilities for planets at relatively long periods. For a photometric monitoring campaign which aims to determine if the planet indeed transits, we calculate the significance of a null result and the subsequent constraints that may be applied to orbital parameters.
\end{abstract}

\section{Transit Probability}

There have been at least five cases in which planetary transits were detected through photometric follow-up of planets already known via their radial velocity (RV) discoveries. The case of HD 17156b (Barbieri et al. 2007) is of particular interest since it is a 21.2 day period planet which happens to have a large eccentricity $(e=0.67)$ and an argument of periastron which places the periapsis of its orbit in the direction toward the observer and close to parallel to the line of sight, resulting in an increased transit probability.

Recent work by Barnes (2007) and Burke (2008) showed that higher eccentricities of planetary orbits will increase their transit probabilities and, consequently, expected yield for transit surveys. We demonstrate the combined effect of the eccentricity, $e$, and argument of periastron, $\omega$, on transit probability. As shown by Kane (2007), the place in a planetary orbit where it is possible for a transit to occur (where the planet passes the star-observer plane perpendicular to the planetary orbit) is when $\omega+f=\pi / 2$. The probability of such a transit occurring, $P_{t}$, is given by

$$
P_{t}=\frac{\left(R_{p}+R_{\star}\right)(1+e \cos (\pi / 2-\omega))}{a\left(1-e^{2}\right)},
$$

where $R_{p}$ and $R_{\star}$ are the radii of the planet and star respectively, and $a$ is the semi-major axis. The orbital configuration, especially with regards to the values of $e$ and $\omega$, plays a major role in determining the likelihood of a planet transiting the parent star.

\section{Argument of Periastron Dependence}

As we rotate the semi-major axis of the orbit around the star we can observe how the transit probability varies using Equation 1.1. This dependence is shown in Figure 1 for eccentricities of 0.3 (dashed line) and 0.6 (dotted line) in comparison with the constant transit probability for a circular orbit (solid line). Since the shape of this variation is independent of period, $P$, the y-axes are scaled for both a 4.0 day and 50.0 day period orbits. Note that $P_{t}$ scales linearly with the sum of $R_{p}$ and $R_{\star}$. 


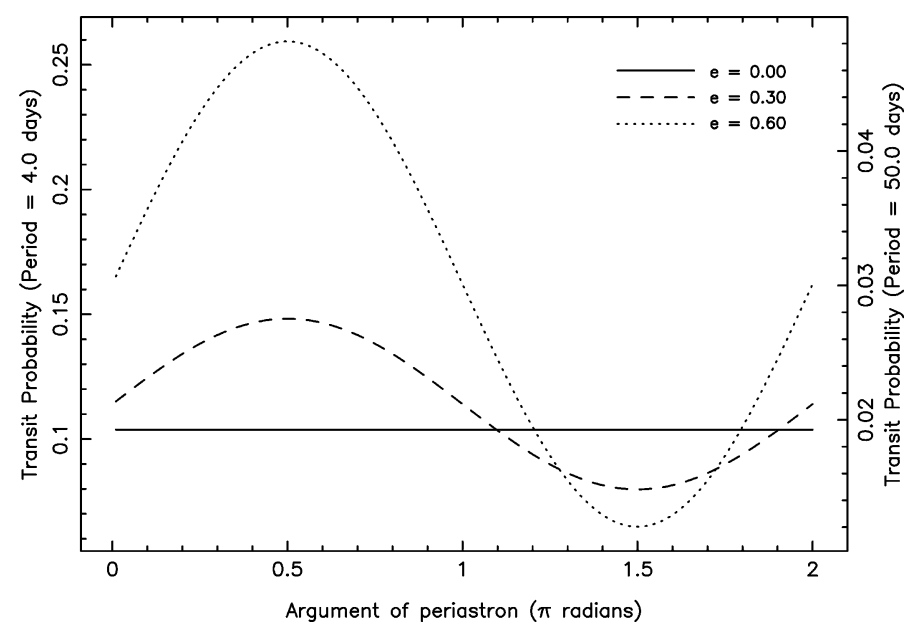

Figure 1. Dependence of geometric transit probability on the argument of periastron, $\omega$, for eccentricities of $0.0,0.3$, and 0.6 , plotted for periods of 4.0 days (left ordinate) and 50.0 days (right ordinate). Stellar and planetary radii are assumed to be a Jupiter and solar radius, respectively.

The peak transit probability occurs at $\omega=\pi / 2$, and the corresponding increase in $P_{t}$ as compared to a circular orbit can be significant; a factor of 1.5 for $e=0.3$ and a factor of 2.5 for $e=0.6$. Moreover, the fraction of the orbital path which produces a higher value of $P_{t}$ than the circular orbit with the same period (corresponding to the fraction of range in $\omega$ for which the dotted or dashed line is above the solid line in Figure 1) increases with increasing eccentricity.

\section{Period Dependence}

The current distribution of eccentricities for the known extra-solar planets indicates that orbits within $0.1 \mathrm{AU}$ tend to be forced into nearly circular orbits through tidal circularization, whereas longer period orbits can possess a great range of eccentricities (Ford \& Rasio 2008). Indeed most of the planets beyond 0.1 AU have eccentricities in excess of 0.3 .

In Figure 2 we show mean transit probability as a function of period after averaging over $0 \leqslant \omega \leqslant 2 \pi$, for the period range $1 \leqslant P \leqslant 50$ days. Eccentricities of $0.0,0.3$, and 0.6 are shown with solid, dashed, and dotted lines, respectively. As expected, we see that doubling the eccentricity from 0.3 to 0.6 creates a significant increase in the mean transit probability. Most affected are the longer period planets whose eccentric orbits can raise their likelihood of transit from a negligible value to a statistically viable number for photometric follow-up.

\section{Application to Known Exoplanets}

Figure 3 shows the transit probability calculated from orbital parameters provided by Butler et al. (2006) for planets with estimates of $e$ and $\omega$ (203 planets in total). For the purposes of comparison, we assume a Jupiter and Solar radius for the values of $R_{p}$ and $R_{\star}$, respectively, and include a solid line which indicates the transit probability for a circular orbit. In addition, the sub-panel in the plot shows the difference in $P_{t}$ between the actual orbit and a hypothetical circular one of the same period (residuals). The mean 


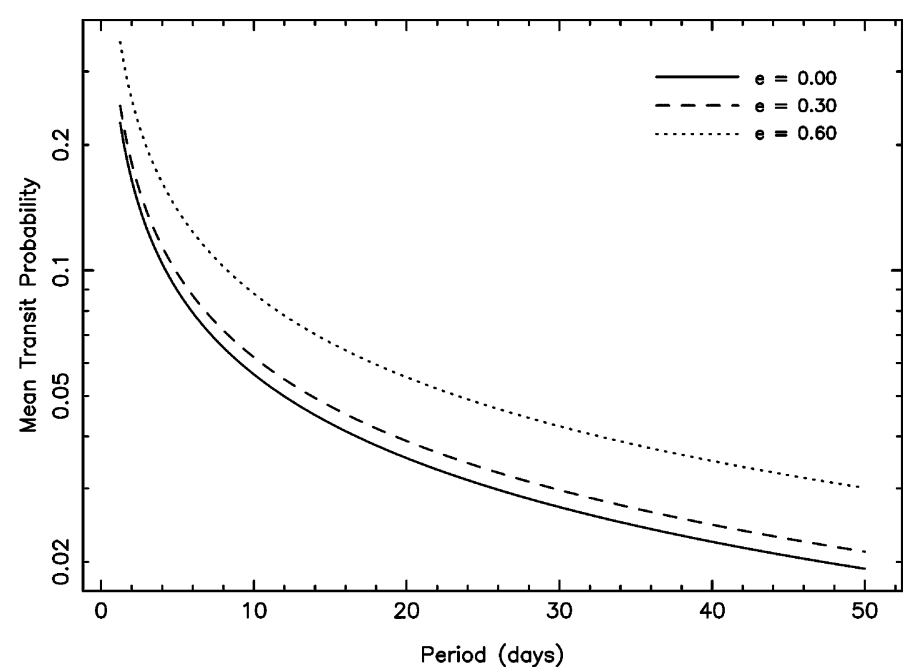

Figure 2. The mean transit probability on a logarithmic scale, averaged over all values of $\omega$, as a function of period, for eccentricities of 0.0, 0.3, and 0.6.

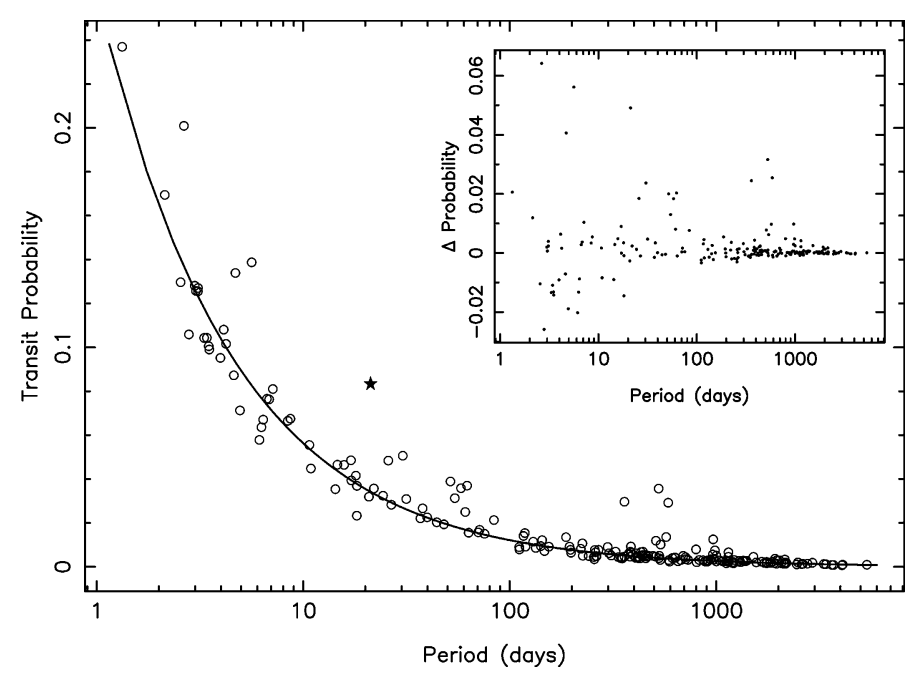

Figure 3. The geometric transit probability for a circular orbit (solid curve) along with the transit probability for $203 \mathrm{RV}$ planets from Butler et al. (2006) calculated from their orbital parameters (open circles). HD $17156 \mathrm{~b}$ is indicated by a 5-pointed star. The sub-panel plots the difference in $P_{t}$ between the the actual orbit and a hypothetical circular orbit for each of the planets.

value of the residuals for all 203 planets is positive but relatively small $\left(4.13 \times 10^{-5}\right)$, and is dominated by the low transit probability of the long period planets. The mean residual of planets with $P<100.0$ days yields an overall increase of $\sim 0.5 \%$ in $P_{t}$.

HD $17156 \mathrm{~b}$, a transiting planet with 21.2 day period, is shown as a 5 -pointed star. Its transit probability is greatly increased by its orbital parameters. Note that the actual $P_{t}$ of HD $17156 \mathrm{~b}$ is larger than the $5 \%$ shown in Figure 3 since the radius of the host star is 1.47 solar radii. At longer periods, the planets with the largest residuals are HD 156846b, HD 4113b, and HD 20782b, which have periods of 359.51, 526.62, and 585.86 days, respectively. The probability residuals for these three planets are 0.024 , 


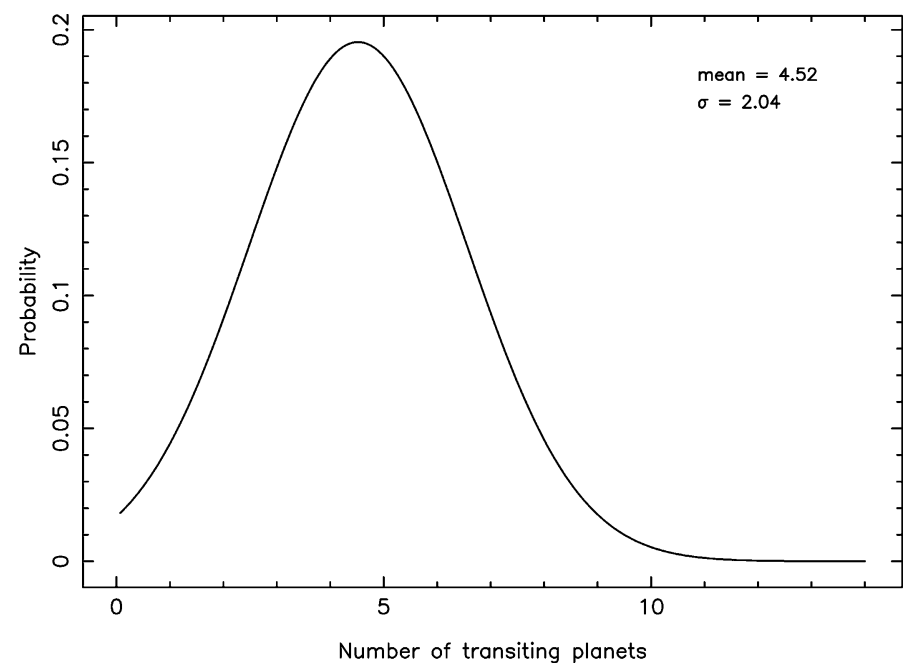

Figure 4. The probability distribution for the 203 planets in the Butler et al. (2006) sample, predicting the number of transiting planets based on their estimated orbital parameters.

0.032 , and 0.025 respectively, the effect of which is to raise their transit probabilities to the same level as HD 17156b if it were in a circular orbit.

\section{Global Statistics and Photometric Follow-up}

The majority of radial velocity planets have been detected around $V<14$ stars. Kane (2007) showed that $1.0 \mathrm{~m}$ class telescopes are ideal instruments to photometrically monitor these targets. By selecting observable targets with well constrained transit windows and high transit probabilities, an optimised campaign can be constructed.

We can determine the significance of a hypothetical null result from a photometric follow-up campaign by performing a Monte-Carlo simulation of the transit probabilities calculated from Equation 1.1 and the tabulated planetary and stellar paramaters of Butler et al. (2006). Performing this calculation 10000 times for each star produces a probability distribution for the number of transiting planets expected from this sample, as shown in Figure 4 . The mean value of $\sim 4.5$ transits peaks at $P_{t} \sim 0.2$ with a standard deviation of $\sim 2.0$. The probability that none of the planets in this sample transit their host star is $\sim 1 \%$. In fact, three of the planets in this sample are known to transit, specifically HD 17156b, GJ 436b, and HD 147506b. Hence the current number of transiting planets from this sample is almost $1 \sigma$ below the expectation. This demonstrates that the offset is quantifiable, and that further transit discoveries in this sample are possible or even likely which would lead to further understanding of the respective observational biases of the RV and transit methods.

\section{References}

Barbieri, M., et al., 2007, A\& A, 476, L13

Barnes, J. W., et al., 2007, PASP, 119, 986

Burke, C. J., 2008, ApJ, 679, 1566

Butler, R. P., et al., 2006, ApJ, 646, 505

Ford, E. B., Rasio, F. A., 2008, ApJ, in press (astro-ph/0703163)

Kane, S. R., 2007, MNRAS, 380, 1488 